\title{
Fatores associados à prática do uso de máscaras pela população paraibana durante a pandemia da COVID-19
}

\section{Factors associated to the practice of using masks by the population of Paraíba during the COVID-19 pandemic}

Factores asociados a la práctica del uso de mascarilla por la población de Paraíba durante la pandemia de COVID-19

Como citar este artigo:

Pereira-Ávila FMV, Lam SC, Gir E, Góes FGB, Freire MEM, Silva ACO. Factors associated to the practice of using masks by the population of Paraíba during the COVID-19 pandemic. Rev Esc Enferm USP. 2021;55:e03735. https://doi.org/10.1590/S1980-220X2020029403735

\section{Fernanda Maria Vieira Pereira-Ávila ${ }^{1}$}

Simon Ching Lam²

Elucir $\mathrm{Gir}^{3}$

Fernanda Garcia Bezerra Góes ${ }^{2}$

Maria Eliane Moreira Freire 4

Ana Cristina de Oliveira e Silva ${ }^{4}$

${ }^{1}$ Universidade Federal Fluminense,

Departamento de Enfermagem,

Rio das Ostras, RJ, Brasil.

${ }^{2}$ Hong Kong Polytechnic University, School of Nursing, Hong Kong, China.

${ }^{3}$ Universidade de São Paulo, Escola de Enfermagem de Ribeirão Preto, Ribeirão Preto, SP, Brasil.

${ }^{4}$ Universidade Federal da Paraíba, Departamento de Enfermagem Clínica, João Pessoa, PB, Brasil.

\section{ABSTRACT}

Objective: To evaluate the practice of using masks by the population of the Brazilian state of Paraíba during the COVID-19 pandemic. Method: Cross-sectional, descriptive-analytical study conducted with adults living in the state of Paraíba through an online instrument from April to May 2020, through the Face Mask Use Scale of Faculdades Metropolitanas Unidas da Paraíba. Results: The participants amounted to $1,307(100.0 \%)$ individuals, who were predominantly female $(78.0 \%)$, aged 35 to 45 $(32.3 \%)$, married $(53.3 \%)$ and post-graduates $(46.9 \%)$. The mean score for the practice of using masks was 18.7 ( $\mathrm{SD}=8.0$; minimum 6.00 ; maximum 30$)$. The use of masks for self-protection scored $9.8(\mathrm{DP}=3.9$; minimum 3.0 ; maximum 15.0$)$, whereas the score for protection of others was $8.9(\mathrm{SD}=4.5$; minimum 3.0 ; maximum 15.0$)$. In the comparison between the scores of the practice of using masks, there was a significant statistical difference for gender, education, age group, and income $(p \leq 0.01)$. The practice of using masks was more frequent in health environments, $7.3(\mathrm{DP}=3.2)$. Conclusion: The practice of using masks was predominant among women, people over 35, married, with an income higher than seven minimum wages, and post-graduation. The use of masks for self-protection was higher than for the protection of others and its use in health environments was higher than in the others.

\section{DESCRIPTORS}

Coronavirus Infections; Pandemics; Masks; Communicable Diseases; Security Measures; Public Health. 


\section{INTRODUÇÃO}

Diante do cenário de pandemia provocado pelo SARSCoV-2 no início de 2020, medidas de proteção individual e coletiva têm sido amplamente divulgadas, com o principal objetivo de controlar a propagação da transmissão do vírus e, consequentemente, da ocorrência da COVID-19. A doença foi detectada pela primeira vez na cidade de Wuhan, China, em dezembro de $2019^{(1-2)}$. A pandemia chegou à América Latina dois meses após a declaração da Organização Mundial da Saúde (OMS), em São Paulo, Brasil, por meio de um homem de 60 anos que viajava pela Itália ${ }^{(3)}$.

Em todo o mundo, até 20 de outubro de 2020, haviam sido confirmados 40.114.293 casos de COVID-19 e 1.114.692 mortes decorrentes. Os Estados Unidos da América apresentavam o maior número de incidência, 8.065.615, seguidos da Índia, Brasil, Rússia e Argentina, respectivamente com 7.597.063, 5.235.344, 1.415.316 e 989.680 casos confirmados $^{(4)}$.

No Brasil, no mesmo período, houve registros, oriundos de todas as regiões e respectivos estados, de aproximadamente 154.176 mortes associadas à COVID-19. A incidência nacional era então de $2.498,6$ casos por 100.000 habitantes. A Região Sudeste registrou, até a mesma data, o maior número de casos (1.837.514) dentre as regiões do Brasil, seguida das Regiões Nordeste, Norte, Sul e Centro-Oeste, respectivamente com 1.424.549, 669.311, 660.908 e 658.445 casos confirmados $^{(5)}$.

A Paraíba era o terceiro estado do Nordeste com a maior taxa de incidência, 3.199,2 casos por 100.000 habitantes, atrás apenas de Sergipe e Piauí, respectivamente com 1.031,1 e 999,1 casos por 100.000 habitantes $^{(5)}$. Compreende-se que a resposta à pandemia não ocorre de modo linear e, desse modo, conhecer o comportamento da população paraibana quanto às medidas de prevenção é a forma mais eficiente até o momento para conter a transmissão e melhorar os indicadores de saúde $\mathrm{e}^{(2,6)}$.

O aumento no número de casos no país tem sido inevitável e vivencia-se o pico da pandemia em várias regiões, inclusive na Paraíba ${ }^{(2,5)}$. Considerando a transmissão do SARS-CoV-2 por via respiratória, o uso de máscaras foi fortemente recomendado não apenas em ambiente hospitalar, mas em comunidades e domicílios ${ }^{(7-9)}$.

Logo, medir a prática do uso de máscaras pela população no período de pandemia da COVID-19 torna-se uma estratégia de prevenção e de intervenção, principalmente nas situações de negligência do uso. A escala FMUS (Face Mask Use Scale) é um instrumento confiável para medir a prática do uso de máscaras, o qual permite identificar os objetivos e circunstâncias de uso. Sua forma de autorrelato facilita a aplicação em estudos populacionais e permite avançar em intervenções educativas e nas medidas preventivas por meio do uso adequado e eficiente de máscaras nas diferentes circunstâncias ${ }^{10)}$.

Tendo em vista que inexistem estudos sobre a temática com a população brasileira até o surgimento da pandemia da COVID-19, entende-se que esta pesquisa é uma das primeiras que apresentam dados sobre a prática do uso de máscaras e fatores associados. Desse modo, o objetivo do presente estudo é avaliar a prática do uso de máscaras pela população paraibana durante a pandemia da COVID-19.

\section{MÉTODO}

\section{TIPO DE ESTUDO}

Trata-se de estudo transversal descritivo-analítico. A pesquisa foi realizada no período de 16 de abril a 15 de maio de 2020, com a participação de indivíduos de várias cidades da Paraíba. Este estudo faz parte do Projeto Multinacional relacionado à prática de utilização de máscara entre o público em geral durante a pandemia da COVID-19 no Brasil.

\section{População}

Para o estudo, foram considerados elegíveis todos os indivíduos maiores de 18 anos de ambos os sexos residentes na Paraíba. Não foram selecionados indivíduos estrangeiros ou paraibanos que estavam temporariamente no Estado.

\section{DefinIÇÃo dA AMOSTRA}

Considerando a população com idade superior a 18 anos de aproximadamente 3.766.528 habitantes e atendendo a um poder estatístico de $80 \%(0,80)$ e nível de significância de $5 \%(0,05)$, a amostra calculada foi de no mínimo 385 participantes. Entretanto, da forma como foi implementada, a fase de coleta resultou em 1.327 participantes. Decidiu-se então considerar esse total de participantes, mantendo o nível de confiança planejado.

\section{Coleta de Dados}

Para a etapa de coleta de dados, uma equipe devidamente capacitada realizou o recrutamento dos indivíduos por meio de mídias digitais (Whatsapp, Facebook, Instagram), com envio de um link para o acesso a documentos virtuais: o Termo de Consentimento Livre e Esclarecido (TCLE) e o formulário da pesquisa. A opção pelo uso de questionário virtual deu-se, principalmente, por proporcionar a participação de indivíduos oriundos de várias cidades da Paraíba, como também por considerar o distanciamento social recomendado no período.

O formulário de coleta de dados contemplou informações gerais e a escala da prática do uso das máscaras faciais (FMUS). Essa escala apresenta propriedades psicométricas satisfatórias ${ }^{(10)}$. Para o estudo no Brasil, a escala foi traduzida e avaliada quanto à validade de face e conteúdo por especialistas na temática e autorizada pelo autor original da escala e coautor do presente estudo ${ }^{(10)}$. Assim, a versão para o português do Brasil (FMU-PB) foi utilizada para este estudo.

A escala é composta por seis itens que discorrem sobre o uso de máscaras nos ambientes públicos, de saúde e domiciliar: 1. Uso de máscara em locais públicos para me proteger 
contra doenças semelhantes à gripe; 2. Uso de máscara em ambientes de saúde para me proteger contra doenças semelhantes à gripe; 3. Uso de máscara em casa quando tenho sintomas de doenças como a gripe; 4. Uso de máscara em locais públicos quando tenho sintomas de doenças como a gripe; 5 . Uso de máscara no serviço de saúde quando tenho sintomas de doenças como a gripe; 6. Uso de máscara em casa quando os membros da família sofrem de doenças como a gripe. A escala constitui-se, ainda, de dois domínios: práticas cautelosas (itens 2, 4 e 5) e práticas negligentes (itens 1, 3 e 6). As opções de respostas variam em uma escala do tipo Likert de cinco pontos, na qual "nunca", "raramente", "às vezes", "frequentemente" e "sempre" representam a prática do uso de máscaras. Uma pontuação de 1 a 5 é designada para opções da escala em ordem crescente ${ }^{(10)}$.

Para a mensuração da prática do uso de máscaras, utiliza-se um escore geral que varia entre 6 e 30, no qual os valores mais altos, mais próximos de 30 , indicam melhor prática de utilização. Para os domínios, o escore varia entre 3 e $15^{(10)}$.

Para este estudo, optou-se por calcular o escore dos itens referentes à prática do uso de máscaras para a autoproteção (itens 1,2 e 6$)$ e proteção do outro $(3,4$ e 5) e nos diferentes ambientes: público (itens 1 e 4), saúde (itens 2 e 5) e domiciliar (itens 3 e 6), com escore variando entre 3 e 10 ${ }^{(10)}$.

\section{ANÁLISE E TRATAMENTO DOS DADOS}

Os dados coletados foram exportados para planilha do programa Microsoft Office Exce ${ }^{\circledR}$ e analisados pelo software $I B M^{\circledR} S P S S$, versão 20.0. Para caracterização sociodemográfica dos participantes, utilizou-se análise estatística descritiva com medidas de frequência absoluta, relativa e de dispersão (desvio-padrão - DP). As variáveis de desfecho (dependentes) foram o escore geral da FMUS e seus domínios. As variáveis independentes foram sexo, faixa etária, nível de instrução, renda familiar, situação conjugal, isolamento social e lavagem das mãos. Para a comparação dos escores da escala entre as variáveis independentes, utilizaram-se testes de hipóteses, a análise de variância (ANOVA) e o Teste $t$ de Student. Foram considerados valores de $\mathrm{p}$-valor $\leq 0,01$ e p-valor $\leq 0,05$.

\section{AspeCtos ÉTIICOS}

O projeto foi aprovado pela Comissão Nacional de Ética em Pesquisa (Conep). Todos os aspectos éticos foram contemplados para sua realização segundo as Resoluções $n^{\circ}$ $466 / 2012$ e no 510/2016.

\section{RESULTADOS}

Participaram do estudo 1.327 (100,0\%) indivíduos da população geral. Houve predomínio de pessoas do sexo feminino, 1.035 (78,0\%), na faixa etária entre 35 e 44 anos, 428 (32,3\%), casadas, 707 (53,3\%), com pós-graduação, 623 $(46,9 \%)$, e renda mensal de sete salários mínimos ou mais, 392 (29,5\%). Com relação às medidas adotadas para evitar a
Tabela 1 - Caracterização dos participantes segundo variáveis individuais e medidas para evitar a COVID-19 - Paraíba, Brasil, 2020.

\begin{tabular}{|c|c|}
\hline Variáveis & n (\%) \\
\hline \multicolumn{2}{|l|}{ Sexo } \\
\hline Masculino & $292(22,0)$ \\
\hline Feminino & $1.035(78,0)$ \\
\hline \multicolumn{2}{|l|}{ Faixa etária (anos) } \\
\hline 18 a 24 & $232(17,5)$ \\
\hline 25 a 34 & $371(28,0)$ \\
\hline 35 a 44 & $428(32,3)$ \\
\hline 45 a 54 & $178(13,4)$ \\
\hline 55 ou mais & $118(8,9)$ \\
\hline \multicolumn{2}{|l|}{ Estado Civil } \\
\hline Solteiro & $535(40,3)$ \\
\hline Casado & $707(53,3)$ \\
\hline Divorciado/separado & $70(5,3)$ \\
\hline Viúvo & $15(1,1)$ \\
\hline \multicolumn{2}{|l|}{ Escolaridade } \\
\hline Fundamental & $7(0,5)$ \\
\hline Médio & $224(16,9)$ \\
\hline Superior & $473(35,6)$ \\
\hline Pós-Graduação & $623(46,9)$ \\
\hline \multicolumn{2}{|l|}{ Renda mensal } \\
\hline$<1$ salário & $47(3,5)$ \\
\hline 1 a 2 salários & $337(25,4)$ \\
\hline 3 a 4 salários & $329(24,8)$ \\
\hline 5 a 6 salários & $214(16,1)$ \\
\hline > 7 salários & $392(29,5)$ \\
\hline Sem rendimentos & $8(0,6)$ \\
\hline \multicolumn{2}{|l|}{ Lavagem das mãos } \\
\hline Não & $33(2,5)$ \\
\hline Sim & $1.294(97,5)$ \\
\hline \multicolumn{2}{|l|}{ Isolamento social } \\
\hline Não & $189(14,2)$ \\
\hline Sim & $1.138(85,8)$ \\
\hline
\end{tabular}

COVID-19, 1.294 (97,5\%) praticavam a lavagem das mãos e $1.138(85,8 \%)$ o isolamento social (Tabela 1$)$.

$\mathrm{Na}$ análise dos itens da FMUS-PB, pode-se perceber que, para o item 1, o qual discorre sobre o uso de máscaras em ambientes públicos, a maior parte dos participantes $(61,1 \%)$ respondeu a opção "sempre". Resultado semelhante foi obtido para o item 2, sobre o uso de máscaras no serviço de saúde, totalizando 65,5\% para a opção "sempre". Por outro lado, para o item 3, acerca do uso de máscaras em casa, apenas 23,8\% relataram realizar essa prática "sempre" (Tabela 2). 
Tabela 2 - Frequência de respostas dos participantes aos itens da versão para o português do Brasil (FMUS-PB) - Paraíba, Brasil, 2020.

\begin{tabular}{|c|c|c|c|c|c|}
\hline & Nunca & Raramente & Às vezes & Frequentemente & Sempre \\
\hline Itens & $\begin{array}{c}n \\
(\%)\end{array}$ & $\begin{array}{c}\mathbf{n} \\
(\%)\end{array}$ & $\begin{array}{c}n \\
(\%)\end{array}$ & $\begin{array}{c}n \\
(\%)\end{array}$ & $\begin{array}{c}\mathbf{n} \\
(\%)\end{array}$ \\
\hline $\begin{array}{l}\text { Eu uso máscara facial em locais públicos } \\
\text { para me proteger contra doenças } \\
\text { semelhantes à gripe. }\end{array}$ & $247(18,6)$ & $\begin{array}{l}79 \\
(6,0)\end{array}$ & $190(14,3)$ & $\begin{array}{c}0 \\
(0,0)\end{array}$ & $811(61,1)$ \\
\hline $\begin{array}{l}\text { Eu uso máscara facial no serviço de } \\
\text { saúde para me proteger contra doenças } \\
\text { semelhantes à gripe. }\end{array}$ & $271(20,4)$ & $\begin{array}{c}64 \\
(4,8)\end{array}$ & $123(9,3)$ & $\begin{array}{c}0 \\
(0,0)\end{array}$ & $869(65,5)$ \\
\hline $\begin{array}{l}\text { Eu uso máscara facial em casa quando } \\
\text { tenho sintomas de doenças como gripe. }\end{array}$ & $706(53,2)$ & $\begin{array}{c}163 \\
(12,3)\end{array}$ & $142(10,7)$ & $\begin{array}{c}0 \\
(0,0)\end{array}$ & $316(23,8)$ \\
\hline $\begin{array}{l}\text { Eu uso máscara facial em locais públicos } \\
\text { quando tenho sintomas de doenças } \\
\text { como gripe. }\end{array}$ & $460(34,7)$ & $\begin{array}{l}103 \\
(7,8)\end{array}$ & $134(10,1)$ & $\begin{array}{c}0 \\
(0,0)\end{array}$ & $630(47,5)$ \\
\hline $\begin{array}{l}\text { Eu uso máscara facial no serviço de } \\
\text { saúde quando tenho sintomas de doenças } \\
\text { como gripe. }\end{array}$ & $389(29,3)$ & $\begin{array}{c}81 \\
(6,1)\end{array}$ & $116(8,7)$ & $\begin{array}{c}0 \\
(0,0)\end{array}$ & $741(55,8)$ \\
\hline $\begin{array}{l}\text { Eu uso máscara facial em casa quando os } \\
\text { membros da família sofrem de doenças } \\
\text { semelhantes à gripe. }\end{array}$ & $747(56,3)$ & $\begin{array}{c}168 \\
(12,7)\end{array}$ & $147(11,1)$ & $\begin{array}{c}0 \\
(0,0)\end{array}$ & $265(20,0)$ \\
\hline
\end{tabular}

Nota: $(\mathrm{n}=1.327)$

O escore geral da prática do uso de máscaras obtido foi $18,7(\mathrm{DP}=8,0)$, variando entre 6 e 30 . Para o domínio práticas cautelosas, obteve-se o escore de $10,5(\mathrm{DP}=4,7)$ e o escore para práticas negligentes foi de $8,2(\mathrm{DP}=3,9)$, sendo o valor mínimo 3 e o máximo 15 . Na avaliação do uso de máscaras para autoproteção, obteve-se o escore de 9,8 ( $\mathrm{DP}=3,9$; mínimo 3,0; máximo 15,0), enquanto o de proteção do outro foi $8,9(\mathrm{DP}=4,5$; mínimo 3,0 ; máximo 15,0$)$. Com relação à prática do uso de máscaras nos ambientes, o escore para ambiente de saúde foi $7,3(\mathrm{DP}=3,2)$, o escore para público foi $6,9(\mathrm{DP}=3,1)$ e domiciliar foi $4,4(\mathrm{DP}=3,0)$.
Na Tabela 3, observam-se o escore geral e os escores médios da FMUS-PB segundo as variáveis individuais e outras medidas de prevenção. Houve diferença significativa na comparação das médias do escore geral entre faixa etária, sexo, escolaridade, estado civil, renda familiar e lavagem das mãos. Os dois domínios apresentaram diferença estatisticamente significativa para as variáveis faixa etária, estado civil e lavagem das mãos. $\mathrm{O}$ domínio que apresentou diferença estatisticamente significativa com o isolamento social foi o domínio prática cautelosa $(\mathrm{p}=0,04)$. Os escores dos itens de autoproteção e proteção do outro também apresentaram diferenças estatisticamente significativas entre faixa etária, estado civil, escolaridade e lavagem das mãos (Tabela 3).

Tabela 3 - Distribuição dos escores da prática do uso de máscaras segundo variáveis individuais e medidas de prevenção para a COVID-19 - Paraíba, Brasil, 2020.

\begin{tabular}{|c|c|c|c|c|c|c|}
\hline Variáveis & f & FMUS & E1 & E2 & E3 & E4 \\
\hline \multicolumn{7}{|l|}{ Faixa etária(2) } \\
\hline 18 a 24 anos & 232 & 16,8 & 9,5 & 7,3 & 8,7 & 8,1 \\
\hline 25 a 34 anos & 371 & 18 & 10,2 & 7,8 & 9,5 & 8,5 \\
\hline 35 a 44 anos & 428 & 19,7 & 11 & 8,7 & 10,2 & 9,5 \\
\hline 45 a 54 anos & 178 & 19,7 & 10,9 & 8,7 & 10,2 & 9,5 \\
\hline 55 anos ou mais & 118 & 19,8 & 10,7 & 9,1 & 10,5 & 9,3 \\
\hline p-valor & & $0,00^{* *}$ & $0,00^{* *}$ & $0,00^{* *}$ & $0,00^{* *}$ & $0,00^{* *}$ \\
\hline \multicolumn{7}{|l|}{$\operatorname{Sexo}^{(1)}$} \\
\hline Masculino & 292 & 17,6 & 9,6 & 8,1 & 9,4 & 8,2 \\
\hline Feminino & 1.035 & 19,0 & 10,8 & 8,3 & 9,0 & 9,1 \\
\hline p-valor & & $0,01^{*}$ & $0,00^{* *}$ & 0,47 & 0,07 & $0,00^{* *}$ \\
\hline
\end{tabular}




\begin{tabular}{|c|c|c|c|c|c|c|}
\hline Variáveis & $\mathrm{f}$ & FMUS & E1 & E2 & E3 & E4 \\
\hline \multicolumn{7}{|l|}{ Escolaridade $^{(2)}$} \\
\hline Fundamental & 7 & 19,4 & 10,1 & 9,3 & 10,7 & 8,7 \\
\hline Médio & 224 & 18 & 10,1 & 7,9 & 9,5 & 8,5 \\
\hline Superior & 473 & 17,8 & 9,8 & 7,9 & 9,4 & 8,4 \\
\hline Pós-Graduação & 623 & 19,7 & 11,2 & 8,5 & 10,2 & 9,5 \\
\hline p-valor & & $0,00^{* *}$ & $0,00^{* *}$ & 0,06 & $0,00^{* *}$ & $0,00^{* *}$ \\
\hline \multicolumn{7}{|l|}{ Estado civil $^{(2)}$} \\
\hline Solteiro & 535 & 17,8 & $9,9^{b}$ & 7,8 & 9,4 & 8,4 \\
\hline Casado & 707 & 19,3 & $10,8^{\mathrm{a}}$ & 8,4 & 10,1 & 9,2 \\
\hline Separado/Divorciado & 70 & 20 & 10,9 & 9,1 & 10,4 & 9,6 \\
\hline Viúvo & 15 & 18,3 & 9,8 & 8,5 & 9,8 & 8,5 \\
\hline $\mathrm{p}$-valor & & $0,00^{* *}$ & $0,00^{* *}$ & $0,01^{*}$ & $0,00^{* *}$ & $0,01^{*}$ \\
\hline \multicolumn{7}{|l|}{ Renda familiar ${ }^{(2)}$} \\
\hline$<1 \mathrm{SM}$ & 47 & 19,3 & 10,2 & 9,1 & 10,5 & 8,8 \\
\hline 1 a 2 SM & 337 & 17,7 & 9,9 & 7,7 & 9,2 & 8,4 \\
\hline 3 a 4 SM & 329 & 18,3 & 10,3 & 7,9 & 9,5 & 8,8 \\
\hline 5 a 6 SM & 214 & 19,2 & 10,8 & 8,4 & 10 & 9,1 \\
\hline $7 \mathrm{SM}$ & 392 & 19,6 & 10,9 & 8,7 & 10,2 & 9,4 \\
\hline Sem renda & 8 & 21,9 & 12,7 & 9,1 & 11,5 & 10,4 \\
\hline p-valor & & $0,01^{* *}$ & 0,06 & $0,00^{* *}$ & $0,00^{* *}$ & 0,06 \\
\hline \multicolumn{7}{|l|}{ Lavagem das mãos ${ }^{(1)}$} \\
\hline Não & 33 & 14,9 & 8,4 & 6,6 & 7,9 & 7 \\
\hline Sim & 1.294 & 18,8 & 10,5 & 8,3 & 9,8 & 8,9 \\
\hline p-valor & & $0,02^{*}$ & $0,02^{*}$ & $0,03 *$ & $0,02^{*}$ & $0,02 *$ \\
\hline \multicolumn{7}{|l|}{ Isolamento social| $^{(1)}$} \\
\hline Não & 189 & 19,2 & 11,1 & 8,1 & 9,9 & 9,2 \\
\hline Sim & 1.138 & 18,7 & 10,4 & 8,2 & 9,8 & 8,9 \\
\hline p-valor & & 0,41 & $0,04 *$ & 0,55 & 0,48 & 0,39 \\
\hline
\end{tabular}

Nota: $(\mathrm{n}=1.327)$

FMUS: Escala da Prática do Uso de Máscaras; E1: Práticas cautelosas; E2: Práticas negligentes; E3: Uso para autoproteção; E4: Uso para proteger o outro. SM: Salário mínimo; Resultados significativos:

* p-valor $\leq 0,05 ; * *$ p-valor $\leq 0,01$

${ }^{1}$ Através do teste t de Student; ${ }^{2}$ Através do teste ANOVA

$\mathrm{Na}$ Tabela 4, referente à prática do uso de máscaras nos ambientes, observa-se diferença estatisticamente significativa entre as variáveis de faixa etária. Os indivíduos entre 18 e 24 anos apresentaram baixos escores de uso para todos os ambientes.
Em relação à variável sexo, as mulheres possuíam maiores escores estatisticamente significativos para uso de máscaras em ambiente de saúde $(\mathrm{p}=0,00)$ e uso de máscaras em ambiente público $(p=0,01)$, mas não para uso de máscaras em ambiente domiciliar $(\mathrm{p}=0,83)$.

Tabela 4 - Distribuição dos escores da prática do uso de máscaras nos ambientes segundo variáveis individuais e medidas de prevenção para a COVID-19 - Paraíba, Brasil, 2020.

\begin{tabular}{|c|c|c|c|c|c|c|c|c|c|c|}
\hline \multirow{2}{*}{ Variáveis } & \multirow{2}{*}{ f } & \multicolumn{3}{|c|}{ E5 } & \multicolumn{3}{|c|}{ E6 } & \multicolumn{3}{|c|}{ E7 } \\
\hline & & Média & DP & $p$ & Média & DP & $p$ & Média & DP & $p$ \\
\hline \multicolumn{11}{|l|}{ Faixa etária ${ }^{(2)}$} \\
\hline 18 a 24 anos & 232 & 6,6 & 3,38 & $0,00^{* *}$ & 4,0 & 2,76 & $0,00^{* *}$ & 6,1 & 3,25 & $0,00^{* *}$ \\
\hline 25 a 34 anos & 371 & 7,3 & 3,19 & & 4,1 & 2,78 & & 6,6 & 3,02 & \\
\hline
\end{tabular}




\begin{tabular}{|c|c|c|c|c|c|c|c|c|c|c|}
\hline \multirow{2}{*}{ Variáveis } & \multirow{2}{*}{ f } & \multicolumn{3}{|c|}{ E5 } & \multicolumn{3}{|c|}{ E6 } & \multicolumn{3}{|c|}{ E7 } \\
\hline & & Média & DP & $p$ & Média & DP & $p$ & Média & DP & $p$ \\
\hline 35 a 44 anos & 428 & 7,3 & 3,06 & & 4,7 & 3,08 & & 7,3 & 3,04 & \\
\hline 45 a 54 anos & 178 & 7,6 & 3,28 & & 4,8 & 3,10 & & 7,3 & 3,20 & \\
\hline 55 anos ou mais & 118 & 7,3 & 3,20 & & 4,9 & 3,20 & & 7,5 & 2,84 & \\
\hline \multicolumn{11}{|l|}{$\operatorname{Sexo}^{(1)}$} \\
\hline Masculino & 292 & 6,7 & 3,33 & $0,00^{* *}$ & 4,4 & 3,04 & 0,83 & 6,5 & 3,16 & $0,01^{*}$ \\
\hline Feminino & 1.035 & 7,5 & 3,15 & & 4,45 & 2,96 & & 7,0 & 3,09 & \\
\hline \multicolumn{11}{|l|}{ Escolaridade $^{(2)}$} \\
\hline Fundamental & 7 & 6,9 & 3,24 & $0,00^{* *}$ & 5,1 & 3,24 & 0,28 & 7,4 & 2,99 & $0,01^{*}$ \\
\hline Médio & 224 & 7 & 3,28 & & 4,2 & 2,91 & & 6,7 & 3,18 & \\
\hline Superior & 473 & 6,8 & 3,38 & & 4,3 & 2,95 & & 6,6 & 3,20 & \\
\hline Pós-Graduação & 623 & 7,9 & 2,97 & & 4,6 & 3,01 & & $7,2^{\mathrm{a}}$ & 3,00 & \\
\hline \multicolumn{11}{|l|}{ 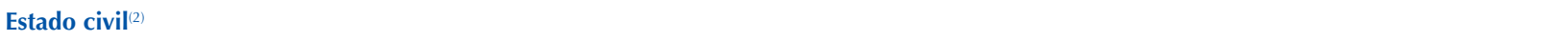 } \\
\hline Solteiro & 535 & 7 & 3,27 & $0,02^{*}$ & 4,3 & 2,89 & 0,25 & 6,5 & 3,16 & $0,00^{* *}$ \\
\hline Casado & 707 & 7,6 & 3,13 & & 4,5 & 2,98 & & 7,2 & 3,08 & \\
\hline Separado/Divorciado & 70 & 7,4 & 3,30 & & 4,9 & 3,45 & & 7,7 & 2,71 & \\
\hline Viúvo & 15 & 6,8 & 3,69 & & 4,3 & 3,24 & & 7,2 & 3,03 & \\
\hline \multicolumn{11}{|l|}{ Renda mensal $^{(2)}$} \\
\hline$<1 \mathrm{SM}$ & 47 & 7 & 3,03 & 0,09 & 5,3 & 2,84 & $0,02^{*}$ & 6,9 & 2,97 & $0,00^{* *}$ \\
\hline 1 a 2 SM & 337 & 7 & 3,19 & & $4^{b}$ & 2,80 & & 6,6 & 3,10 & \\
\hline 3 a 4 SM & 329 & 7,2 & 3,31 & & 4,4 & 2,85 & & 6,7 & 3,20 & \\
\hline 5 a $6 \mathrm{SM}$ & 214 & 7,5 & 3,08 & & 4,5 & 3,07 & & 7,1 & 3,02 & \\
\hline $7 \mathrm{SM}$ ou mais & 392 & 7,6 & 3,24 & & $4,7^{\mathrm{a}}$ & 3,15 & & 7,3 & 3,06 & \\
\hline Sem renda & 8 & 9 & 1,51 & & 4,9 & 2,30 & & 8,0 & 3,02 & \\
\hline \multicolumn{11}{|l|}{ Lavagem das mãos $^{(1)}$} \\
\hline Não & 33 & 6,2 & 3,62 & 0,08 & 3,8 & 2,86 & 0,17 & 5,0 & 3,34 & $0.00^{* *}$ \\
\hline Sim & 1.294 & 7,3 & 3,20 & & 4,4 & 2,98 & & 7,0 & 3,09 & \\
\hline \multicolumn{11}{|l|}{ Isolamento social $^{(1)}$} \\
\hline Não & 189 & 7,9 & 2,91 & $0,00^{* *}$ & 4,4 & 2,90 & 0,81 & 6,9 & 3,12 & 0,75 \\
\hline Sim & 1.138 & 7,2 & 3,25 & & 4,4 & 2,99 & & 6,9 & 3,11 & \\
\hline
\end{tabular}

Nota: $(\mathrm{n}=1.327)$

SM: Salário mínimo; E5: Uso em ambiente de saúde; E6: Uso em domicílio; E7: Uso em local público Resultados significativos: * p-valor $\leq 0,05 ;{ }^{* *}$ p-valor $\leq 0,01$

$\mathrm{Na}$ Figura 1, referente à distribuição do escore geral e dos demais escores da FMUS-PB, o domínio práticas cautelosas e o uso para autoproteção apresentaram os maiores valores de medianas (11), enquanto o uso em ambiente domiciliar apresentou menor valor de mediana (3). Observa-se que a média, sinalizada no gráfico como ponto vermelho, e a mediana apresentaram valores próximos. 


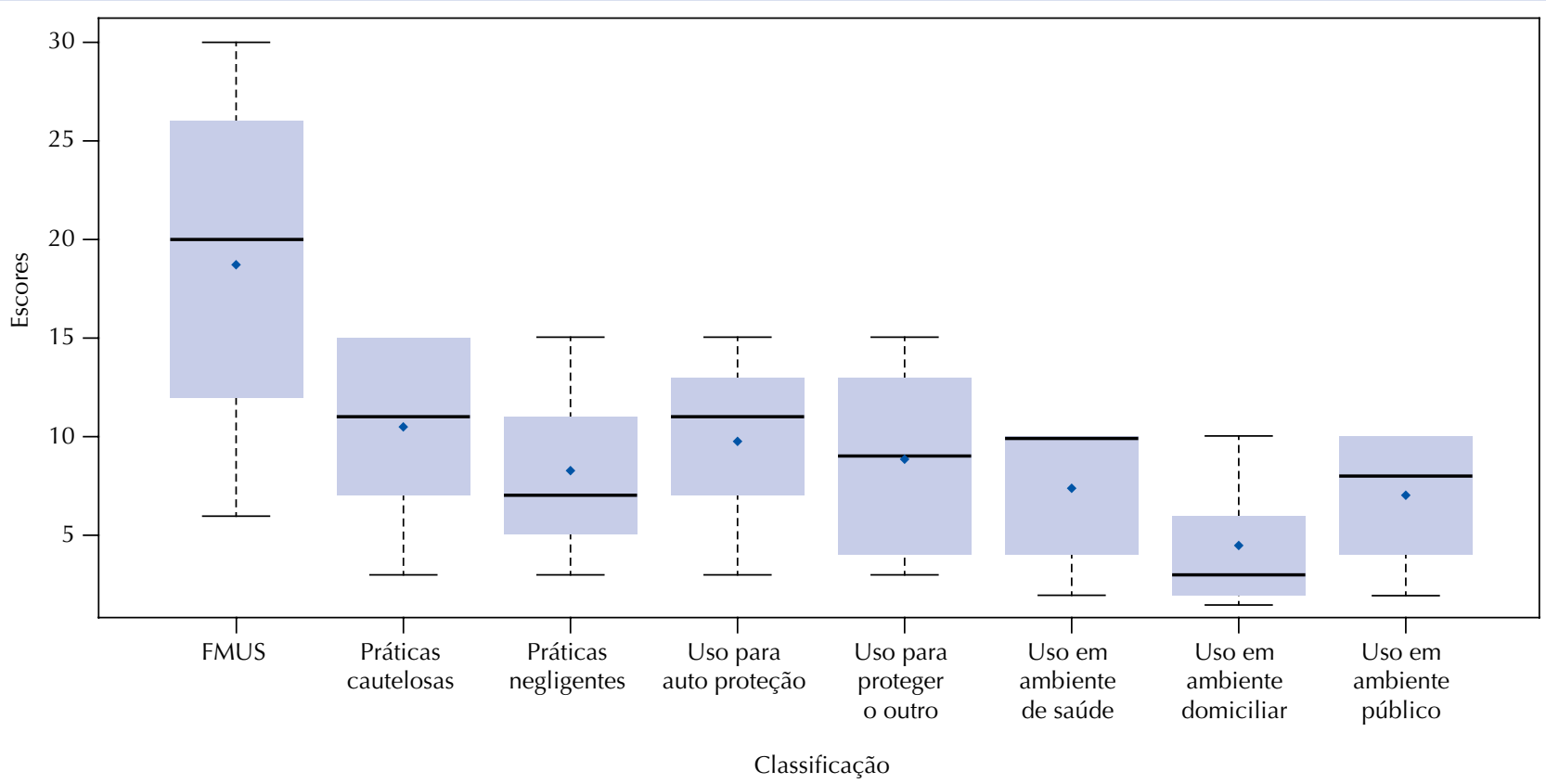

Nota: $(\mathrm{n}=1.327)$

Figura 1 - Boxplot dos escores da prática do uso de máscaras do escore geral e dos demais itens da escala FMUS-PB - Paraíba, Brasil, 2020.

\section{DISCUSSÃO}

Este estudo avaliou a prática do uso de máscaras entre a população da Paraíba considerando práticas cautelosas e negligentes, uso para autoproteção e proteção do outro nos ambientes público, de saúde e domiciliar.

Em relação à caracterização sociodemográfica dos participantes, a população constitui-se em grande parte por mulheres adultas e com maior escolaridade e renda mensal acima de sete salários mínimos. Esse achado é condizente com um estudo desenvolvido em Hong Kong com 1.020 indivíduos sobre conhecimento, atitudes e práticas em relação às medidas de proteção (uso de máscaras, lavagem das mãos, distanciamento social) quanto à infecção respiratória (A/H7N9), com predomínio de mulheres adultas de maior escolaridade ${ }^{(11)}$.

A prática do uso de máscaras pela população estudada obteve resultados satisfatórios. Percebe-se que a prática do uso de máscaras neste estudo foi maior quando comparada à de uma pesquisa desenvolvida no Egito, onde menos da metade da população estudada usava máscaras, apesar de mais da metade dessa população acreditar que a prática do uso protege o indivíduo de doenças de transmissão respiratória, configurando uma prática negligente ${ }^{(12)}$. A prática do uso de máscaras, etiqueta respiratória, higienização das mãos e medidas de distanciamento social são importantes na supressão da COVID-19(2,11,13).

A prática negligente quanto ao uso de máscaras pode estar relacionada à falta de consenso das autoridades sanitárias e dos governos quanto à determinação ou obrigatoriedade do uso ${ }^{(14)}$. No Brasil, a determinação do uso de máscaras pelo público em geral vem acontecendo em períodos distintos em cada região a partir do comportamento epidemiológico da COVID-19 e conforme a realidade de cada estado e município, segundo recomendação do Ministério da Saúde ${ }^{(2)}$. Neste estudo, os adultos jovens apresentaram menores escores quanto às práticas negligentes do uso de máscaras quando comparados aos escores dos adultos mais velhos.

Recomendações distintas podem ter um efeito negativo e favorecer a prática negligente quanto ao uso de máscaras e outras medidas preventivas. Em alguns países, tem-se observado, nos veículos de comunicação e mídias sociais, a disseminação de notícias falsas relacionadas à COVID-19 e medidas de proteção e prevenção (incluindo tipo e uso de máscaras). Tal fato se agrava com a falta de conhecimento e discernimento da população sobre o que pode ser verdadeiro e adequado, sobretudo quando se trata de algo novo e ainda desconhecido ${ }^{(15-16)}$.

Neste estudo, observa-se uma importante associação quanto às medidas de prevenção da COVID-19, pois os indivíduos que higienizavam as mãos apresentaram maior escore na escala da prática do uso de máscaras. Estudos de revisão sistemática e meta-análise mostram a eficácia significativa da combinação da prática do uso de máscaras e a lavagem das mãos contra o vírus da influenza em comunidades $^{(17-18)}$. Entretanto, a complexidade que envolve tais medidas de prevenção é real, podendo muitas vezes estar relacionada a fatores como o comportamento humano, compensação de percepção de risco, subestimação da responsabilidade individual e falta de conhecimento, atitudes que podem interferir na adesão às medidas de prevenção( ${ }^{(7,19-21)}$.

Evidências científicas sobre a eficiência do uso de máscaras pela população em período de pandemias por doenças respiratórias são escassas e contestadas ${ }^{(22)}$. Porém, diante da pandemia da COVID-19, deve-se aplicar o princípio da precaução e incentivar a população quanto à prática do uso de máscaras. 
Ressalta-se que o período de coleta de dados do presente estudo refere-se ao momento após o decreto do Ministério da Saúde sobre o uso de máscaras de tecido pela população em ambientes públicos ${ }^{(8)}$. A prática do uso de máscaras pela população brasileira não era comum e acredita-se que nunca havia sido vivenciada ${ }^{(2)}$, o que pode comprometer a adoção dessa medida de precaução.

$\mathrm{Na}$ ausência de estudos que possam subsidiar a discussão desta pesquisa quanto à prática do uso de máscaras pela população brasileira, foram consideradas pesquisas realizadas em outros países e cidades, principalmente estudos em países onde a prática do uso de máscaras tem sido frequente diante de epidemias de transmissão respiratória anteriores, como os da Âsia ${ }^{(2)}$.

A população do estudo era mais propensa a usar máscaras para se proteger em vez de proteger o outro e mais propensa a usar máscaras em ambientes públicos do que em ambientes de saúde ou em casa. Estudo realizado em Hong Kong com 399 entrevistados identificou que os indivíduos eram mais propensos a usar máscaras em ambiente de saúde e para proteger os outros do que para se protegerem ${ }^{(23)}$.

Por conseguinte, a prática do uso de máscaras deve acontecer dentro e fora do ambiente domiciliar, tanto para proteger-se como para proteger o outro, visando à prevenção do contato respiratório com desconhecidos assintomáticos ou oligossintomáticos ${ }^{(24)}$. Ademais, pessoas saudáveis se infectam em locais públicos ${ }^{(25)}$.

Chama atenção a baixa frequência quanto à prática do uso de máscaras em ambiente doméstico. $\mathrm{O}$ ambiente domiciliar é percebido como seguro, independentemente da presença ou não de sintomas respiratórios da gripe ${ }^{(10)}$. No entanto, indivíduos sintomáticos respiratórios devem utilizar máscaras em ambiente doméstico. A transmissão dentro das famílias continua sendo uma preocupação, uma vez que $66 \%$ dos casos confirmados diagnosticados em Hong Kong foram disseminados entre os membros da família ${ }^{(18)}$. Existem vantagens potenciais quanto ao uso de máscaras em ambientes públicos e de saúde, seja por indivíduos sintomáticos ou não, como a redução do risco potencial de exposição, também observada nos ambientes domiciliares ${ }^{(18)}$.

De um modo geral, a prática do uso de máscaras e a frequência de uso nos diferentes ambientes para a população do estudo foram consideradas boas, exceto no ambiente domiciliar; porém, ressalta-se que essa prática deve ser adotada por toda população. Acredita-se que, no momento da coleta de dados, algumas dificuldades, como a ausência de teste rápido para o rastreamento de casos, o cenário político inamistoso para decisões baseadas em evidências e as divergências de informações e recomendações entre governo, estados e munícipios, parecem ter influenciado a adoção de medidas adequadas de proteção e precaução para a COVID-19(26). No entanto, a percepção de aumento de risco desempenha um papel importante no uso de máscaras ${ }^{(19)}$. $\mathrm{O}$ período de execução da pesquisa correspondia ao pico da pandemia e acredita-se que o uso de máscaras pela população do estudo tenha aumentado.

Recomenda-se que as máscaras sejam utilizadas em conjunto com outras medidas não farmacológicas de prevenção, como distanciamento social, boa etiqueta respiratória e higiene regular das mãos. Além do mais, o uso de máscaras pela população poderá transmitir uma falsa sensação de segurança, induzindo o indivíduo a desvalorizar o conjunto de medidas de prevenção diante da pandemia da COVID-19 $9^{(27-28)}$.

Os achados deste estudo levantam pontos importantes para reflexões acerca das medidas de proteção contra a COVID-19 adotadas pela população, as quais devem ser incorporadas e implementadas por meio da educação permanente. A formação permanente possibilita o desenvolvimento dos profissionais, especialmente da equipe de enfermagem, favorecendo as demandas dos serviços de saúde da população e dos trabalhadores diante da prática do uso de máscaras, considerando que se trata de algo novo e que exige conhecimentos baseados em evidências científicas para que sejam incorporadas em sua prática ações de educação em saúde efetivas e eficazes, conforme as demandas identificadas na população diante do panorama epidemiológico vigente.

Algumas limitações deste estudo devem ser elencadas: a exclusão dos analfabetos digitais, o impedimento do auxílio ao participante diante da incompreensão de alguma pergunta e a impossibilidade do conhecimento quanto às circunstâncias em que o questionário foi respondido.

\section{CONCLUSÃO}

Os resultados evidenciam que a maior parte dos participantes deste estudo realizou a prática do uso de máscaras, que foi superior entre mulheres, pessoas acima de 35 anos, casadas, com renda mensal acima de sete salários e com pós-graduação. $\mathrm{O}$ uso de máscaras para autoproteção foi maior do que para a proteção do outro e a utilização em ambientes de saúde foi maior do que nos demais ambientes. No entanto, deve-se considerar que o uso de máscaras pela população só é eficaz se a utilização for satisfatória e seu uso for associado a outras medidas preventivas para evitar a COVID-19. Desse modo, torna-se premente a realização de estudos que possam demonstrar fatores que influenciam a prática do uso de máscaras e que permitam identificar as relações de causa e efeito para prevenção e controle da COVID-19.

\section{RESUMO}

Objetivo: Avaliar a prática do uso de máscaras pela população paraibana durante a pandemia da COVID-19. Método: Estudo transversal descritivo-analítico realizado com adultos residentes no estado da Paraíba via instrumento online, no período de abril a maio de 2020, por meio da escala da prática do uso de máscaras das Faculdades Metropolitanas Unidas da Paraíba. Resultados: Participaram do estudo $1.307(100,0 \%)$ indivíduos, com predominância do sexo feminino (78,0\%), faixa etária entre 35 e 45 anos (32,3\%), casados $(53,3 \%)$ e com pós-graduação (46,9\%). O escore médio da prática do uso de máscaras foi de 18,7 (DP = 8,0; mínimo 6,00; máximo 30). A utilização de máscaras para autoproteção obteve o escore de 9,8 ( $\mathrm{DP}=3,9$; mínimo 3,0; máximo 15,0), enquanto o escore de proteção do outro foi 8,9 (DP = 4,5; mínimo 3,0; máximo 15,0). Na comparação entre os escores da prática do uso de máscaras, houve diferença estatística entre sexo, escolaridade, faixa etária e renda $(\mathrm{p} \leq 0,01)$. A prática de utilização de máscaras foi maior em ambientes de saúde $7,3(\mathrm{DP}=3,2)$. Conclusão: A prática do uso de máscaras predominou entre mulheres, pessoas acima de 35 anos, casadas, com renda 
acima de sete salários mínimos e com pós-graduação. O uso de máscaras para autoproteção foi maior do que para a proteção do outro e a utilização em ambientes de saúde foi maior do que nos demais.

\section{DESCRITORES}

Infecções por Coronavírus; Pandemias; Máscaras; Doenças Transmissíveis; Medidas de Segurança; Saúde Pública.

\section{RESUMEN}

Objetivo: Evaluar la práctica del uso de mascarilla por la población de Paraíba durante la pandemia de COVID-19. Método: Estudio descriptivo-analítico transversal realizado con adultos residentes en el estado de Paraíba vía instrumento online, en el período de abril a mayo de 2020, mediante la escala de la práctica del uso de la máscara de Faculdades Metropolitanas Unidas da Paraíba. Resultados: Participaron en el estudio un total de 1.307 (100,0\%) personas, predominantemente del sexo femenino (78,0\%), en el grupo de edad de 35 a 45 años (32,3\%), casadas $(53,3 \%)$ y con postgrado (46,9\%). La puntuación media de la práctica del uso de la mascarilla fue de 18,7 $(\mathrm{SD}=8,0$; mínimo 6,00; máximo 30). El uso de mascarillas para la autoprotección obtuvo una puntuación de 9,8 $(\mathrm{SD}=3,9 ;$ mínimo 3,0; máximo 15,0), mientras que la puntuación para la protección de los demás fue de 8,9 ( $\mathrm{SD}=4,5 ;$ mínimo 3,0; máximo 15,0). En la comparación entre las puntuaciones de la práctica del uso de la mascarilla, hubo una diferencia estadística entre el sexo, la educación, el grupo de edad y los ingresos ( $\mathrm{p} \leq 0,01)$. La práctica del uso de la mascarilla fue mayor en las áreas de salud: 7,3 $(\mathrm{SD}=3,2)$. Conclusión: La práctica del uso de mascarillas predominó entre las mujeres, las personas mayores de 35 años, casadas, con ingresos superiores a siete salarios mínimos y con postgrado. El uso de las mascarillas para la autoprotección fue mayor que para la protección del otro y el uso en áreas de salud fue mayor que en las otras áreas.

\section{DESCRIPTORES}

Infecciones por Coronavirus; Pandemias; Máscaras; Enfermedades Transmisibles; Medidas de Seguridad; Salud Pública.

\section{REFERÊNCIAS}

1. European Centre for Disease Prevention and Control. Situation update worldwide, as of 2 april 2020 [Internet]. 2020 [cited 2020 Apr 2]. Available from: https://www.ecdc.europa.eu/en/geographical-distribution-2019-ncov-cases

2. Croda J, Oliveira WK, Frutuoso RL, Mandetta LH, Baia-da-Silva DC, Brito-Sousa JD, et al. COVID-19 in Brazil: advantages of a socialized unified health system and preparation to contain cases. Rev Soc Bras Med Trop. 2020;53:e20200167. https://doi.org/10.1590/0037-86820167-2020

3. Rodriguez-Morales AJ, Gallego V, Escalera-Antezana JP, Méndez CA, Zambrano LI, Franco-Paredes C, et al. COVID-19 in Latin America: the implications of the first confirmed case in Brazil. Travel Med Infect Dis. 2020;29:101613. https://doi.org/10.1016/j.tmaid.2020.101613

4. World Health Organization. Coronavirus disease (COVID-19) Dashboard [Internet]. Geneva: WHO; 2020 [cited 2020 Oct 20 ]. Available from: https://covid19.who.int/

5. Brasil. Ministério da Saúde. Secretarias Estaduais de Saúde. Painel Coronavírus [Internet]. Brasília, DF: Ministério da Saúde; 2020 [cited 2020 out. 20]. Available from: https://covid.saude.gov.br/

6. Lodge EK, Schatz AM, Drake JM. Protective population behavior change in outbreaks of emerging infectious disease. bioRxiv. [Preprint]. 2020. https://doi.org/10.1101/2020.01.27.9215367.

7. Garcia LP. Use of facemasks to limit COVID-19 transmission. Epidemiol Serv Saúde. 2020;29(2):e2020023. https://doi.org/10.5123/ s1679-49742020000200021

8. Brasil. Ministério da Saúde. Nota informativa nº 3/2020-CGPAP/DESF/SAPS/MS. Brasília, DF: Ministério da Saúde; 2020 [cited 2020 Oct 20]. Available from: http://portal.antaq.gov.br/wp-content/uploads/2020/04/1586014047102-Nota-Informativa.pdf

9. Center for Disease Prevention and Control. Recommended guidance for extended use and limited reuse of N95 filtering facepiece respirators in healthcare settings [Internet]. 2020 [cited 2020 June 15]. Available from: https://www.cdc.gov/niosh/topics/hcwcontrols/ recommendedguidanceextuse.html\#note2

10. Lam SC, Chong ACY, Chung JYS, Lam MY, Cham LM, Shum CY, et al. Methodological study on the evaluation of face mask use scale among public adult: cross-language and psychometric testing. Korean J Adult Nurs. 2020;32(1):46-56. https://doi.org/10.7475/kjan.2020.32.1.46

11. Chan EY, Cheng CK, Tam G, Huang Z, Lee P. Knowledge, attitudes, and practices of Hong Kong population towards human A/H7N9 influenza pandemic preparedness, China, 2014. BMC Public Health. 2015;15:943. https://doi.org/10.1186/s12889-015-2245-9

12. Abdelhafiz AS, Mohammed Z, Ibrahim ME, Ziady HH, Alorabi M, Ayyad M, et al. Knowledge, perceptions, and attitude of Egyptians towards the Novel Coronavirus Disease (COVID-19). J Community Health. 2020 [Preprint]. https://doi.org/10.1007/s10900-020-00827-7

13. Cowling BJ, Ali ST, Ng TWY, Tsang TK, Li JCM, Fong MW, et al. Impact assessment of non-pharmaceutical interventions against coronavirus disease 2019 and influenza in Hong Kong: an observational study. Lancet Public Health. 2020;5(5):e279-88. https://doi.org/10.1016/ S2468-2667(20)30090-6

14. Cheng VCC, Wong SC, Chuang VW, So SY, Chen JH, Sridhar S, et al. The role of community-wide wearing of face mask for control of coronavirus disease 2019 (COVID-19) epidemic due to SARS-CoV-2. J Infect. 2020;81(1):107-14. https://doi.org/10.1016/j.jinf.2020.04.024

15. Shimizu K. 2019-nCoV, fake news, and racism. Lancet. 2020;395(10225):685-6. https://doi.org/10.1016/S0140-6736(20)30357-3

16. Hernández-García I, Giménez-Júlvez T. Assessment of Health Information About COVID-19 Prevention on the Internet: Infodemiological Study. JMIR Public Health Surveill. 2020;6(2):e18717. https://doi.org/10.2196/18717

17. Wong VW, Cowling BJ, Aiello AE. Hand hygiene and risk of influenza virus infections in the community: a systematic review and meta-analysis. Epidemiol Infect. 2014;142(5):922-32. https://doi.org/10.1017/s095026881400003x

18. Cheng VCC, Wong SC, Chen JHK, Yip CCY, Chuang VWM, Tsang OTY, et al. Escalating infection control response to the rapidly evolving epidemiology of the coronavirus disease 2019 (COVID-19) due to SARS-CoV-2 in Hong Kong. Infection Control \& Hospital Epidemiology. 2020;41:493-8. https://doi.org/10.1017/ice.2020.58 
19. Howard J, Huang A, Li Z, Tufekci Z, Zdimal V, Van Der WH, et al. Face masks against COVID-19: an evidence review. PNAS. 2020 Oct 30. https://doi.org/10.20944/preprints202004.0203.v1

20. Oliveira AC, Coaglio LT, Iquiapaza RA. What has the covid-19 pandemic taught us about adopting preventive measures? Texto Contexto Enferm. 2020;29:e20200106. https://doi.org/10.1590/1980-265x-tce-2020-0106

21. Brasil. Ministério da Saúde. Máscaras caseiras podem ajudar na prevenção contra o coronavírus [Internet]. Brasília, DF: Ministério da Saúde; 2020 [cited 2020 Apr 7]. Disponível em: https://www.saude.gov.br/noticias/agencia-saude/46645-mascaras-caseiras-podemajudarna-prevencao-contra-o-coronavirus

22. Greenhalgh T, Schmid MB, Czypionka T, Bassler D, Gruer L. Face masks for the public during the covid-19 crisis. BMJ. $2020 ; 369: m 1435$. https://doi.org/10.1136/bmj.m1435

23. Ho HSW. Use of face masks in a primary care outpatient setting in Hong Kong: Knowledge, attitudes and practices. Public Health. 2012;126(12):1001-6. https://doi.org/10.1016/j.puhe.2012.09.010

24. Spellberg B, Haddix M, Lee R, Wu-Butler S, Holtom P, Yee H, et al. Community prevalence of SARS-CoV-2 among patients with influenza like illnesses presenting to a Los Angeles Medical Center in March 2020. JAMA. 2020;323(19):1966-7. https://doi.org/10.1001/jama.2020.4958

25. Ferretti L, Wymant C, Kendall M, Zhao L, Nurtay A, Abeler-Dörner L, et al. Quantifying SARS-CoV-2 transmission suggests epidemic control with digital contact tracing. Science. 2020;368(6491):eabb6936. https://doi.org/10.1126/science.abb6936

26. Cimerman S, Chebabo A, Cunha CA, Rodríguez-Morales AJ. Deep impact of COVID-19 in the healthcare of Latin America: the case of Brazil. Braz J Infect Dis. 2020;24(2):93-5. https://doi.org/10.1016/j.bjid.2020.04.005

27. Mueller W, Horwell CJ, Apsley A, Steinle S, McPherson S, Cherrie JW, et al. The effectiveness of respiratory protection worn by communities to protect from volcanic ash inhalation. Part I: filtration efficiency tests. Int J Hyg Environ Health. 2018; 221(6):967-76. https://doi.org/10.1016/j.ijheh.2018.03.012

28. Shakya KM, Noyes A, Kallin R, Peltier R. Evaluating the efficacy of cloth facemasks in reducing particulate matter exposure. J Expo Sci Environ Epidemiol. 2017;27:352-7. https://doi.org/10.1038/jes.2016.42 